\title{
Avaliação da competição no mercado de carbono: elaboração e validação de questionário
}

\author{
Camila TORRES $^{\text {; }}$ Ricardo K.S. FERMAM² \\ $\triangle$ cmagtorres@gmail.com \\ 1. ABNT - Associação Brasileira de Normas Técnicas, Rua Conselheiro Nébias, 1131, São Paulo, Brasil. \\ 2. Instituto Nacional de Metrologia, Qualidade e Tecnologia, Coordenação Geral de Acreditação, Rua Santa \\ Alexandrina, 416, Rio de Janeiro, Brasil.
}

Histórico do Artigo:

Recebido: 22 de junho de 2016

Aceito: 26 de outubro de 2016

Publicado: 22 de dezembro de 2016

Resumo: 0 objetivo do presente artigo é apresentar o desenvolvimento e a validação de questionário para determinação das forças competitivas atuantes no mercado de validação de projetos de gases de efeito estufa. 0 questionário foi elaborado com questões fechadas, dispostas em núcleos temáticos e direcionado a coletar dados que apontem as forças competitivas e o grau de competição do mercado voluntário de validação de projetos de gases de efeito estufa. 0 questionário foi devidamente validado, utilizando-se a determinação do coeficiente alfa de Cronbach para verificação da confiabilidade interna. Os resultados obtidos na etapa de validação revelam que o questionário elaborado pode ser utilizado como instrumento da pesquisa para o qual foi originalmente proposto. Palavras-chave: Estratégia, Forças Competitivas, Gases de Efeito Estufa.

\section{Competition assessment in carbon market: questionnaire development and validation}

\begin{abstract}
The aim of this paper is to present the development and validation of a questionnaire to determine the competitive forces operating in the validation market of greenhouse gas projects. The questionnaire was prepared with closed questions, arranged in thematic groups and targeted to collect evidences that indicate the competitive forces and the competition degree of the voluntary market of validation of greenhouse gas projects. The questionnaire was validated using the determination of Cronbach's alpha for verification of internal reliability. The results obtained in the validation step reveals that the questionnaire prepared can be used as a research tool for which it was originally proposed.
\end{abstract}

Keywords: Strategy, Competitive Forces, Greenhouse Gases. 


\section{Evaluación de la competencia en el mercado de carbono: elaboración y validación del cuestionario}

Resumen: El objetivo de este trabajo es presentar el proceso de elaboración y validación de un cuestionario que determine las fuerzas competitivas que operan en el mercado de validación de proyectos de gases de efecto invernadero. El cuestionario fue preparado con preguntas cerradas, dispuestas en grupos temáticos y dirigidas para recoger pruebas que indiquen las fuerzas competitivas y el grado de competencia del mercado voluntario de validación de proyectos de gases de efecto invernadero. El cuestionario fue validado, mediante la determinación del alfa de Cronbach para la verificación de la fiabilidad interna. Los resultados obtenidos en la etapa de validación revelan que el cuestionario preparado se puede utilizar como una herramienta de investigación para la que se propuso originalmente.

Palabras clave: Estrategia, Fuerzas Competitivas, Gases de Efecto Invernadero.

\section{INTRODUÇÃO}

0 mercado de validação de projetos de gases de efeito estufa ${ }^{1}$ é composto por empresas, genericamente denominadas organismos de validação e verificação (OVV), que atuam na avaliação objetiva da exatidão e integridade dos projetos, elaborados por empresas ou organizações, quanto ao atendimento aos requisitos de validação expressos em programas ${ }^{2}$ específicos. Tal atuação por parte desses organismos é dita como sendo de terceira parte, por sua independência e imparcialidade com relação às empresas ou organizações que elaboram os projetos, guardando-se entre elas, contudo, uma relação comercial quanto ao serviço de validação prestado, competindo entre si pela conquista de market share.

Segundo Porter (1986), na luta pela fatia de mercado, a competição não se manifesta apenas na figura dos outros participantes, mas encontra-se firmada na sua economia subjacente e algumas forças competitivas vão bem além dos players nela estabelecidos. 0 estado da competição de um setor depende de cinco forças básicas - a ameaça de novos entrantes, rivalidade entre concorrentes existentes, pressão por produtos/serviços substitutos, poder de negociação dos compradores e o poder de negociação dos fornecedores (Porter, 1986). A análise

\footnotetext{
${ }^{1}$ Define-se "projetos de gases de efeito estufa" como sendo as atividades que alteram as condições identificadas no cenário de referência (linha de base), causando redução de emissões de gases de efeito estufa ou melhoria na remoção de gases de efeito estufa. (ABNT, 2007).

${ }^{2}$ Sistema ou programa internacional, nacional ou regional, voluntário ou obrigatório, que registra, contabiliza, ou administra as emissões, as remoções, as reduções de emissões de gases de efeito estufa (GEE) ou as melhorias nas remoções de gases de efeito estufa fora da organização ou projeto de GEE. (ABNT, 2007).
} 
das forças competitivas da indústria onde estão inseridos os organismos de validação e verificação permite que a empresa:

\begin{abstract}
Encontre uma posição dentro do mercado de atuação em que possa se defender contra forças competitivas externas, influenciando-as a seu favor, bem como, permite observar oportunidades e ameaças latentes ou remotas. Esta análise também proporciona a observância de forças e fraquezas no ambiente interno que poderão impactar favoravelmente ou não na capacidade competitiva da empresa (Wittmann et al, 2011, p.10).
\end{abstract}

Objetivo do presente artigo é apresentar o desenvolvimento e a validação de questionário para determinação das forças competitivas atuantes no mercado de validação de projetos de gases de efeito estufa. A relevância do estudo reside no fato de os dados obtidos através do questionário apontar as forças competitivas e o grau de competição que venham a contribuir para a proposição de estratégias competitivas genéricas visando a sustentabilidade em longo prazo do mercado de empresas validadoras de projetos de gases de efeito estufa. Este trabalho pode ser considerado inédito para o setor de validação de projetos de GEE, bem como para os estudos de competitividade, por não terem sido identificados trabalhos com este enfoque até 0 momento.

\title{
MATERIAL E MÉTODOS
}

\section{Elaboração do questionário}

0 questionário foi composto de questões fechadas, dispostas em núcleos temáticos e direcionado a coletar dados que apontem as forças competitivas e o grau de competição do mercado voluntário de validação de projetos de gases de efeito estufa. Na Tabela 1 estão explicitados os núcleos, suas denominações, siglas e objetivos e definições.

As questões foram inicialmente extraídas a partir dos seguintes trabalhos: Medeiros (2007) e Olinger (2011) e então adaptadas para o escopo deste trabalho. 
Tabela 1 - Núcleos (N) temáticos do questionário

Núcleo 1 (Nl) - Característica do serviço prestado pela Empresa

Objetivo/Definição: Que definem o tipo de serviço prestado pela empresa.

Núcleo 2 (N2) - Atitude da Empresa referente aos custos

Objetivo/Definição: Refere-se à atitude da empresa com relação aos seus custos para disponibilização do serviço no mercado frente aos custos dos concorrentes.

Núcleo 3 (N3) - Atitude da Empresa referente ao preço

Objetivo/Definição: Competição de preços

Núcleo 4 (N4) - Formação de Barreiras de Entrada

Objetivo/Definição: Uma das formas de impedir a chegada de um novo entrante são as barreiras de entrada estabelecidas pelas empresas já inseridas nesta indústria.

Núcleo 5 (N5) - Diferencial em relação aos serviços

Objetivo/Definição: A diferenciação de serviço vai requerer das entrantes, potenciais valores elevados, quanto aos gastos de marketing, em busca da fidelidade dos consumidores das firmas já atuantes nesta indústria.

Núcleo 6 (N6) - Custos de Mudança

Objetivo/Definição: São custos com que defronta o comprador quando muda de um fornecedor para outro.

Núcleo 7 (N7) - Canais de Distribuição

Objetivo/Definição: Quando o concorrente acaba de se estabelecer deve garantir meios de distribuir seu novo serviço.

Núcleo 8 (N8) - Rivalidade entre as Empresas existentes

Objetivo/Definição: Destaca as disputas por fatias de mercado onde as empresas sentem a necessidade de otimizar suas posições frente aos concorrentes.

Núcleo 9 (N9) - Ameaças de Substitutos

Objetivo/Definição: Serviços que podem substituir ou serem utilizados na mesma função em determinada indústria.

Núcleo 10 (N10) - Poder de Negociação dos Compradores

Objetivo/Definição: 0 poder de negociação dos compradores se manifesta na capacidade dos mesmos em pressionar a redução dos preços da indústria, buscar mais qualidade ou mais serviços e jogar os concorrentes uns contra os outros.

Núcleo 11 (N11) - Poder de Negociação dos Fornecedores

Objetivo/Definição: 0 poder de negociação dos fornecedores se manifesta na capacidade dos mesmos de majorar os preços ou reduzir a qualidade dos bens e serviços fornecidos.

Fonte: TORRES, 2016. 


\section{Elaboração do instrumento de validação}

Dentre os vários aspectos críticos que compõem o campo da elaboração de questionários o mais fundamental está relacionado ao conhecimento da validade e confiabilidade dos instrumentos (DUTRA, 2004).

Os estudos de validade determinam se o questionário está realmente medindo aquilo a que se propôs medir. Todos os estudos de validade, independente do tipo, conduzem ao mesmo resultado: o grau de confiança que pode ser colocado nas inferências feitas a partir dos escores obtidos pelas escalas ou pelos questionários (STREINER et al, 2014). Dentre os diferentes tipos de validade, este trabalho se foca em uma validação de conteúdo. A validade de conteúdo indica se os itens do questionário abrangem adequadamente todos os aspectos importantes e relevantes do problema que está sendo investigado.

A validação de conteúdo do questionário foi realizada de maneira independente por especialistas previamente identificados e selecionados que procederam a uma avaliação crítica deste, e consequentemente dos critérios que orientaram a sua construção. Foi usado como critério para identificação e seleção dos especialistas o conhecimento na temática da pesquisa (competição e validação de projetos de emissões de gases de efeito estufa). Cada especialista recebeu um protótipo do questionário de avaliação das forças competitivas e do grau de competição do mercado voluntário de validação de projetos de GEE; e um instrumento de validação cujas respostas constituem o registro do seu julgamento.

A metodologia utilizada para validação de conteúdo do questionário demandou a elaboração de um instrumento para julgamento deste, direcionado aos especialistas, com 0 objetivo de operacionalizar a avaliação dos núcleos e suas respectivas questões. As questões do instrumento de validação foram inicialmente extraídas a partir do trabalho de Roiseman (2008) e então adaptadas para o escopo deste. Inicialmente 0 instrumento dos especialistas avaliou as questões contidas nos núcleos do questionário e no final foi feita uma avaliação do questionário globalmente.

Todo instrumento usado para avaliação crítica de estudos deve sistematizar critérios de julgamento (OXMAN E GUYATT, 1991), em uma sequência de itens de fácil compreensão, apresentados de forma breve, clara e com vocábulos apropriados, para que as respostas obtidas 
possuam maior fidedignidade. 0 julgamento foi baseado nos atributos constantes da Tabela $2 \mathrm{e}$ Tabela 3.

Tabela 2 - Atributos avaliados na validação por núcleo do questionário

\begin{tabular}{l}
\hline Relevância: grau de importância/necessidade \\
\hline Abrangência: grau de cobertura da dimensão \\
\hline Clareza: qualidade do que é claro ou inteligível \\
\hline $\begin{array}{l}\text { Decisões subjetivas: julgamento por critérios não } \\
\text { explícitos }\end{array}$ \\
\hline Possibilidade de gerar vieses: desvios induzidos pela \\
formulação da questão \\
\hline Redundância: grau de repetição das questões \\
\hline Extensão do núcleo: suficiência do tamanho \\
\hline Ambiguidade: possibilidade de gerar dúvidas \\
\hline
\end{tabular}

Fonte: TORRES, 2016.

Tabela 3 - Atributos avaliados na validação global do questionário

\begin{tabular}{l}
\hline Aplicabilidade \\
\hline Relevância \\
\hline Abrangência \\
\hline Extensão \\
\hline Possibilidade de sucesso \\
\hline Nota para os núcleos \\
\hline
\end{tabular}

Fonte: TORRES, 2016.

Para avaliação foi elaborada uma escala tipo Likert que estabelece uma correspondência entre os graus de avaliação/julgamento do atributo e uma escala numérica variando de 1 a 5 , onde a escolha de maior pontuação corresponde ao melhor julgamento.

A escala Likert consiste tipicamente de um conjunto de palavras e números que podem expressar graus de concordância, aceitabilidade, probabilidade, similaridade com os enunciados que podem ser perguntas, ou afirmações sobre o objeto, ou a própria mensuração de um atributo (ROISEMAN, 2008). 
Neste trabalho foi solicitado a cada especialista escolher uma alternativa de resposta que correspondesse ao grau de avaliação em relação ao conteúdo.

Numa escala tipo Likert as alternativas são normalmente em número de cinco. Cerca da metade das opções deve ser positiva e outra metade, negativa. Na avaliação de atitudes uma escolha é positiva, quando o consentimento com o seu conteúdo corresponde a posições favoráveis; é negativo, se o consentimento com o seu conteúdo corresponde a posições desfavoráveis. Na atribuição de pontos às respostas assinaladas, o valor de cada enunciado deve ser considerado. Para itens positivos, a alternativa que expressa maior grau de concordância deve receber maior escore e a que expressa maior grau de discordância o menor escore.

Após a pontuação cada especialista pôde comentar questões e itens que deveriam ser revistos, sugerir questões não contempladas, identificar vieses, relacionar itens redundantes, indicar questões com direcionamento e as ambíguas, pôde também relacionar as falhas de cada núcleo e do questionário globalmente.

\section{Validação do questionário}

0 questionário foi encaminhado para 15 especialistas, selecionados por julgamento ${ }^{3}$ (amostragem não probabilística), dos quais retornaram 8 instrumentos de validação respondidos, representando 53\% do total de material que foi enviado para validação, conforme pode ser visto na Tabela 4. A equipe que participou das avaliações foi constituída por: cinco (05) ambientalistas, membros dos organismos de verificação e validação (0VV), membros do organismo de acreditação e membro de renomada empresa de consultoria, todos atuantes no mercado e três (03) economistas, professores doutores da Universidade Federal do Rio de Janeiro (UFRJ).

0 erro aleatório está presente em qualquer mensuração científica e acaba por reduzir a precisão das mesmas. Desta forma, a confiabilidade refere-se à precisão da mensuração independente do que é medido (NUNNALLY, 1978). A confiabilidade retrata o quanto os valores observados estão associados aos valores verdadeiros. Todavia, em diversas situações reais, como

\footnotetext{
${ }^{3} \mathrm{Na}$ amostragem por julgamento, o pesquisador usa o seu julgamento para selecionar os membros da população que são boas fontes de informação precisa (OLIVEIRA, 2001). É usada quando não se conhecem o tamanho do universo e os indivíduos são selecionados através de critérios subjetivos do pesquisador (MAROTTI et al, 2008).
} 
os valores verdadeiros são desconhecidos, não é possível definir a confiabilidade pelo cálculo da correlação entre os valores observados e verdadeiros.

Tabela 4 - Distribuição dos especialistas que participaram das etapas de validação do questionário

\begin{tabular}{ccc}
\hline Especialistas & Enviados & Respondidos \\
\hline Ambientalistas & 9 & 5 \\
Economistas & 6 & 3 \\
\hline Total & 15 & 8 \\
\hline
\end{tabular}

Fonte: TORRES, 2016.

De acordo com Trochim (2001), a consistência interna refere-se à intensidade com que os itens de um questionário estão correlacionados entre si e com o resultado total da pesquisa, o que demonstra uma medida da confiabilidade do mesmo. Em especial, este trabalho dedicase à verificação da confiabilidade do questionário através da utilização de um dos métodos estatísticos mais comumente utilizados para medição da consistência interna - 0 coeficiente alfa de Cronbach, apresentado por Lee J. Cronbach em 1951.

Visto que todos os itens de um questionário usam a mesma escala de avaliação, o coeficiente $\alpha, \operatorname{com} \alpha \in[0,1]$, é calculado a partir da variância dos itens e das covariâncias entre os itens através da seguinte equação (equação l):

$$
\alpha=\left(\frac{k}{k-1}\right)\left(1-\frac{\sum_{i=1}^{k} S_{i}^{2}}{S_{t}^{2}}\right)
$$

Onde: k é o número de itens do questionário, $S_{l}^{2}$ é a variância do item i e $S_{t}^{2}$ é a variância total do questionário.

Assim, o coeficiente $\alpha$ pode ser determinado da seguinte maneira: em primeiro lugar, calcula-se a variância de cada coluna, denotada por $S_{l}^{2}$ e, depois se soma todas estas variâncias. Em seguida, deve-se obter a soma total das notas atribuídas por cada avaliador e, 
posteriormente calcular a variância destas somas. A Tabela 5 ilustra a aplicação passo a passo do coeficiente, onde cada coluna indica um item, cada linha um avaliador, e o encontro entre um item e um avaliador $\left(\mathrm{X}_{\mathrm{nk}}\right)$ indica a resposta deste avaliador para este item, dentro da escala (DA HORA, 2010).

Tabela 5 - Matriz de julgamentos dos n avaliadores à luz dos k itens do questionário

\begin{tabular}{cccccccc}
\cline { 2 - 6 } & \multicolumn{7}{c}{ Itens } \\
\hline Avaliadores & $\mathbf{1}$ & $\mathbf{2}$ & $\ldots$ & $\boldsymbol{i}$ & $\ldots$ & $\boldsymbol{k}$ & Soma ou Total \\
\hline $\mathbf{1}$ & $X_{11}$ & $X_{12}$ & $\ldots$ & $X_{1 i}$ & $\ldots$ & $X_{1 k}$ & $X_{1}$ \\
$\mathbf{2}$ & $X_{21}$ & $X_{22}$ & $\ldots$ & $X_{2 i}$ & $\ldots$ & $X_{2 k}$ & $X_{2}$ \\
$\vdots$ & $\vdots$ & $\vdots$ & $\vdots$ & $\vdots$ & $\vdots$ & $\vdots$ & $\vdots$ \\
$p$ & $X_{p 1}$ & $X_{p 2}$ & $\ldots$ & $X_{p i}$ & $\ldots$ & $X_{p k}$ & $X_{p}$ \\
$\vdots$ & $\vdots$ & $\vdots$ & $\vdots$ & $\vdots$ & $\vdots$ & $\vdots$ & $\vdots$ \\
$\boldsymbol{n}$ & $X_{n 1}$ & $X_{n 2}$ & $\ldots$ & $X_{n i}$ & $\ldots$ & $X_{n k}$ & $X_{n}$ \\
\hline
\end{tabular}

Fonte: CRONBACH, 2004.

\section{RESULTADOS E DISCUSSÃ0}

\section{Análise da confiabilidade de consistência interna}

Nesta seção apresentam-se os resultados da análise da consistência interna dos itens pertencentes a cada um dos núcleos empregados.

Segundo cada um dos onze núcleos, o questionário apresenta confiabilidade distinta, conforme pode ser visto na Tabela 6. Neste caso, conforme Freitas (2005), o cálculo de uma confiabilidade única para o questionário (envolvendo todos os itens) não se justifica, uma vez que 0 valor de tal confiabilidade seria maior do que a confiabilidade de um ou mais núcleos. Além do que, vale destacar que não se pode afirmar que um núcleo tem maior ou menor confiabilidade que outra, pois estes possuem número de itens distintos.

Nunnally (1978) recomenda um nível mínimo de 0,7 para o alfa de Cronbach. Hair et al (2005) comentam que para pesquisas exploratórias, o alfa pode ser reduzido para 0,6. A Tabela 3 apresenta os alfas dos núcleos. Os núcleos apresentaram valores de alfas maiores do que o recomendado por ambos os autores, com exceção do primeiro núcleo que apresentou um valor de alfa bem próximo de 0 (zero). 
Tabela 6 - Análise da confiabilidade de consistência interna dos itens do questionário

\begin{tabular}{ccc}
\cline { 2 - 2 } & Coef. Alfa de Cronbach \\
\hline Núcleos & $\propto$ \\
N1 & 0,0857 \\
N2 & 0,9490 \\
N3 & 0,9269 \\
N4 & 0,9435 \\
N5 & 0,9326 \\
N6 & 0,9618 \\
N7 & 0,9035 \\
N8 & 0,9500 \\
N9 & 0,9423 \\
N10 & 0,9211 \\
N11 & 0,9507 \\
\hline
\end{tabular}

Fonte: TORRES, 2016 .

0 modelo para estimação do alfa de Cronbach é válido para $\{\alpha \in \Re \mid-\infty<\alpha \leq 1 \wedge \alpha \neq 0\}$, mas deve ser interpretado no intervalo entre 0 e 1 , onde os valores negativos do alfa devem ser considerados como escalas sem confiança (NICHOLS, 1999).

Segundo a literatura científica existente, inúmeros fatores que podem intervir na confiabilidade de questionários (tanto positivamente quanto negativamente), dentre os quais, destaca-se que segundo Hayes (1995), a amostra de avaliadores semelhantes pode resultar em um questionário de baixa confiabilidade. Se na avaliação de um item todos os avaliadores consideram 5 (pressupondo uma escala que varia de 1 a 5), não há variância neste item e, portanto, a confiabilidade calculada terá valor zero.

No entanto, é importante ressaltar que os avaliadores geralmente possuem compreensões e interpretações diferentes sobre os itens em julgamento. Deste modo, considerando uma amostra relevante de avaliadores, é muito improvável que haja um consentimento absoluto a respeito da avaliação de determinado item. Entretanto, quando os avaliadores são próximos ou equivalentes em formação profissional, provavelmente haverá menor variabilidade nas análises, o que pode levar a menores valores de confiabilidade do questionário. 


\section{Adequação do questionário após validação e versão final}

0 núcleo 1 por corresponder a menos de 10\% do questionário total, não possuir questões relevantes e ter tido um alfa baixo, não foi mantido na versão final do questionário. Com relação aos demais núcleos, as questões que os compõem foram readequadas conforme comentários dos especialistas consultados, à luz dos requisitos das Tabelas 2 e 3 . A versão final do questionário, após a etapa de validação, encontra-se na Tabela 7.

Tabela 7 - Questionário para Análise da Competição de 0VV

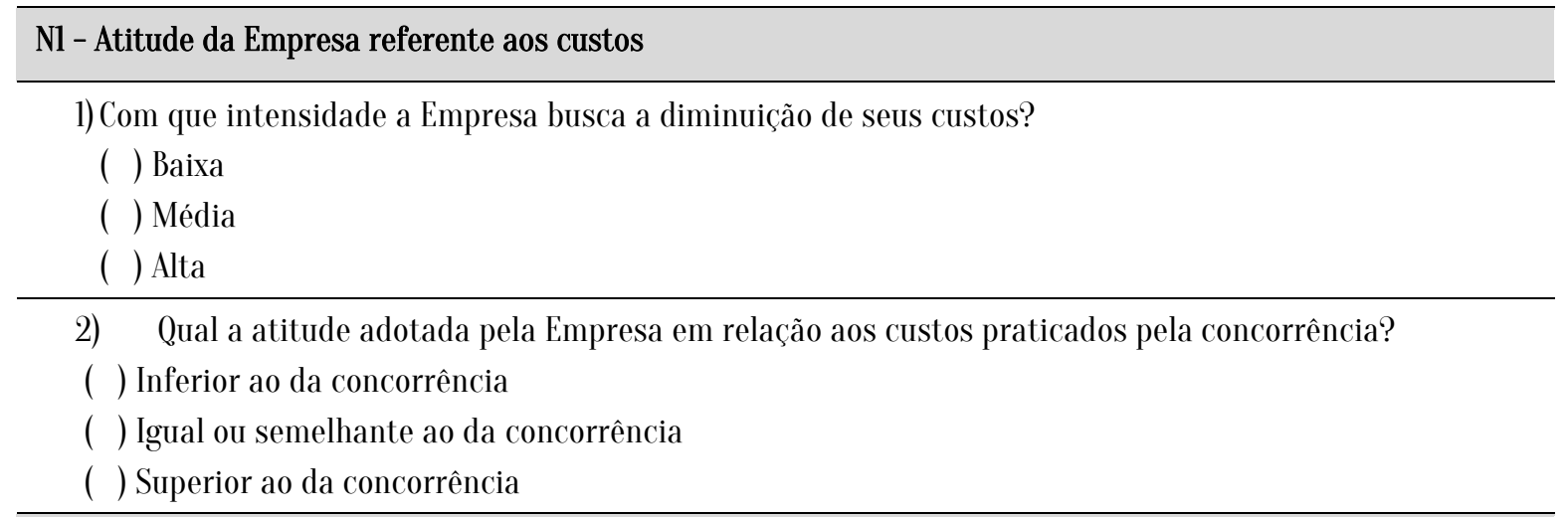

\section{N2 - Atitude da Empresa referente ao preço}

3) 0 preço do serviço é constituído baseado em qual situação?

( ) Equivalente à média do mercado

( ) Equivalente aos custos da Empresa

( ) Outros. Especificar:

4) 0 s preços em comparação ao mercado são:

( ) Inferior ao do mercado

( ) Igual ou semelhante ao do mercado

( ) Superior ao do mercado

\section{N3 - Formação de Barreiras de Entrada}

5) A Empresa sente-se ameaçada com a possível entrada de novos concorrentes no mercado?

( ) Sim, constantemente

( ) Pouco preocupa

( ) Não há preocupação

6) A Empresa se preocupa em ter uma diferenciação da sua marca?

( ) Sim

( ) Não

7) Se sim, este diferencial tem dificultado a entrada de novos concorrentes?

( ) Sim

( ) Não

8) A Empresa procura identificar sua marca junto ao consumidor através de quais meios?

( ) Mídias impressas, faladas e televisivas 
\begin{tabular}{l}
\hline ( ) Mídia próxima ao mercado em que atua (Outdoor, Banner, placas) \\
( ) Marketing exclusivo (interno) aos clientes consumidores \\
\hline 9) O investimento necessário para ingressar neste mercado pode dificultar a entrada de concorrentes? \\
( ) Dificulta \\
( ) Pouco dificulta, pois o investimento não é muito grande \\
( ) Não dificulta
\end{tabular}

10) 0 investimento inicial é considerado como barreira de entrada?

( ) Sim

( ) Não

11) Os serviços ofertados pela Empresa têm dificultado a entrada de novos concorrentes?

( ) Sim

( ) Pouco provável

( ) Não

\section{N4 - Diferencial em relação aos serviços}

12) Qual o investimento necessário para iniciar no mercado?

( ) Abaixo de R\$50.000,00

( ) De R\$50.000,00 a R\$100.000,00

( ) De $\mathrm{R} \$ 100.000,00$ a $\mathrm{R} \$ 250.000,00$

( ) De $\mathrm{R} \$ 250.000,00$ a $\mathrm{R} \$ 500.000,00$

( ) Acima de $\mathrm{R} \$ 500.000,00$

13) Em relação ao faturamento, qual a média de investimentos em marketing ambiental necessários para fixação da marca?
( ) Até $1 \%$
( ) Entre 1\% e 5\%
( ) Mais de $5 \%$
( ) Pouco expressivo quase nulo

\section{N5 - Custos de Mudança}
14) Qual o principal custo enfrentado pela Empresa?
( ) Custos com a acreditação
( ) Custos com a qualificação dos auditores/validadores
( ) Custos com treinamento de funcionários
( ) Outros. Quais?

\section{N6 - Canais de Divulgação}

15) A Empresa realiza investimentos em marketing ambiental com qual (is) objetivo (s)?

( ) Para promover a venda dos principais serviços

( ) Para promover a marca da Empresa

( ) Para promover a venda dos serviços com fixação de marca

( ) Outro. Especifique:

16) Qual o percentual da Empresa em investimento em marketing ambiental para diferenciar sua marca?

( ) Até $1 \%$

( ) Entre $1 \%$ e $5 \%$

( ) Mais de $5 \%$

( ) Não faz 


\section{N7 - Rivalidade entre as Empresas existentes}

17) Como sua Empresa se apresenta ao mercado em relação aos concorrentes?

( ) Pequeno porte

( ) Médio porte

( ) Grande porte

18) Qual o principal item presente na rivalidade com os concorrentes?

( ) Preço dos serviços

( ) Diferenciação dos serviços

( ) Outros. Quais?

19) Qual a principal dificuldade encontrada pela Empresa no mercado?

( ) Concorrência com empresas maiores

( ) Proximidade regional dos concorrentes

( ) Preços oferecidos pelos concorrentes

( ) Outros. Quais?

20) Em relação ao mercado, qual o crescimento da Empresa?

( ) Até $3 \%$

( ) Entre $3 \%$ e $5 \%$

( ) Mais de $5 \%$

( ) Mantém-se estabilizada

21) Quanto aos custos fixos, a Empresa considera que tem:

( ) Altos custos fixos

( ) Médios custos fixos

( ) Baixos custos fixos

\section{N8 - Ameaças de Substitutos}

22) Existem concorrentes no mercado com serviços substitutos aos oferecidos pela sua Empresa?

( ) Sim

( ) Não

( ) Não Sei

23) Qual a estratégia que a Empresa utiliza para fidelizar os clientes diante dos serviços substitutos oferecidos pela concorrência?
( ) Preço
( ) Diferencial nos serviços
( ) Outros. Quais?

N9 - Poder de Negociação dos Compradores

24) Qual a importância dos serviços prestados pela sua Empresa para o cliente?

( ) Essencial

( ) Muito importante

( ) Pouco importante

\section{N10 - Poder de Negociação dos Fornecedores}

25) De uma forma global, o principal fornecedor (auditor/validador) tem poder no mercado?

( ) Sim, totalmente

( ) Sim, pouca, mas tem

( ) Não tem 
26) Há existência de custos para troca de auditor/validador? E estes são significativos?

( ) Sim e relativamente altos

( ) Sim e relativamente baixos

( ) Sim em alguns casos

( ) Não em caso algum

27) Há fidelidade a um único ou poucos auditores/validador?

( ) Sim e com exclusividade

( ) Sim e sem exclusividade

( ) Sim em alguns casos

( ) Não em caso algum

Fonte: TORRES, 2016.

\section{CONCLUSÃ0}

0 mercado de carbono, no que se refere aos projetos de GEE, é algo ainda pouco estudado no que se refere à intensidade da competição entre seus players, em especial a dos organismos de verificação e validação. Neste sentido, o presente questionário se constitui numa ferramenta de pesquisa importante.

Apesar do bom resultado obtido com a validação do questionário, indicado pelo alto valor de alfa obtido, as perguntas podem ser aprimoradas no sentido de possibilitar uma interpretação mais objetiva dos possíveis resultados. Não obstante, é possível refazer o questionário em futuras pesquisas, de forma a haver maior número de questões para cada núcleo estabelecido, observando-se nesse caso o propósito das questões não tenderem para uma resposta.

Com relação à diferenciação, por exemplo, as empresas de um modo geral se acham diferenciadas e dificilmente admitem que apenas o preço possa ser o fator de decisão. As respostas obtidas podem, deste modo, convergir para qualidade e diferencial. Assim, os resultados devem ser analisados criticamente, corrigindo-os com o próprio conhecimento do pesquisador acerca dos organismos estudados.

\section{REFERÊNCIAS BIBLIOGRÁFICAS}

ABNT - ASSOCIAÇ̃̃O BRASILEIRA DE NORMAS TÉCNICAS. ABNT NBR ISO 14064 - Gases de efeito estufa - Parte 3: Especificação e orientação para a validação e verificação de declarações relativas a gases de efeito estufa. Rio de Janeiro, 2007. 
CRONBACH, Lee J.; SHAVELSON, Richard J. My current thoughts on coefficient alpha and successor procedures. Educational and psychological measurement, v. 64, n. 3, p. 391-418, 2004.

DA HORA, Henrique Rego Monteiro; MONTEIR0, Gina Torres Rego; ARICA, José. Confiabilidade em questionários para qualidade: um estudo com o Coeficiente Alfa de Cronbach. Produto \& Produção, v. 11, n. 2, p. 85-103, 2010.

DUTRA, M.V.P. Redes neurais artificiais no reconhecimento e classificação do transtorno do déficit da atenção e hiperatividade. Tese de Doutorado. Engenharia Biomédica - COPPE/ Universidade Federal do Rio de Janeiro, 2004.

FREITAS, André Luís Policani; RODRIGUES, Sidilene Gonçalves. A avaliação da confiabilidade de questionários: uma análise utilizando o coeficiente alfa de Cronbach. Anais do Simpósio de Engenharia de Produção-SIMPEP, Bauru, São Paulo, v. 12, 2005.

HAIR, J., ANDERSON, R.E., TATHAM, R.L., BLACK, W.C. Multivariate data analysis. 4th ed. Englewood Cliffs: Prentice Hall, 1995.

HAYES, B. E., Medindo a satisfação do cliente, Rio de Janeiro: Editora Qualitymark, 228p. 1995. H0SS, Marcelo; TEN CATEN, Carla Schwengber. Processo de Validação Interna de um Questionário em uma Survey Research sobre ISO 9001: 2000.Produto \& Produção, v. 11, n. 2, p. 104-119, 2010.

MAR0TTI, Juliana et al. Amostragem em pesquisa clínica: tamanho da amostra. Revista de Odontologia da Universidade Cidade de São Paulo, v. 20, n. 2, p. 186-194, 2008.

MEDEIROS, Edsel. Estratégias competitivas no comércio varejista de combustíveis, segundo o modelo de Porter. Monografia. Universidade Federal de Santa Catarina, 2007.

NICHOLS, David P. My coefficient alpha is negative. SPSS Keywords, v. 68, p. 1-4, 1999.

NUNNALLY, J. Psychometric Theory (2nd eel.). New York, Iv \cGrow-Hill, 1978.

OLINGER, Fabio. A Gestão Estratégica: Análise das Forças Competitivas de Porter no ramo de clínicas de estética. Monografia. Universidade Federal do Paraná, 2011.

OLIVEIRA, Tânia Modesto Veludo de. Amostragem não Probabilística: Adequação de Situações para uso e Limitações de amostras por Conveniência, Julgamento e Quotas. Administração on line, v. 2, n. 3, 2001.

OXMAN, Andrew D.; GUYATT, Gordon H. Validation of an index of the quality of review articles. Journal of clinical epidemiology, v. 44, n. 11, p. 1271-1278, 1991.

PORTER, Michael E. Estratégia competitiva: técnicas para análise de indústrias e da concorrência. Campus, 1986.

ROISEMAN, Maria de Marilacc Lima et al. Elaboração e validação de um questionário para avaliar conhecimento de pediatras e médicos de família na prevenção da doença cardiovascular na infância. Dissertação de Mestrado. Instituto Fernandes Figueira, Rio de Janeiro, 2008.

STREINER, David L.; NORMAN, Geoffrey R.; CAIRNEY, John. Health measurement scales: a practical guide to their development and use. Oxford University Press, USA, 2014.

TORRES, Camila Maria Aguiar. 0 mercado voluntário de carbono no Brasil: oportunidades de negócios para empresas de validação de projetos de gases de efeito estufa. Dissertação de Mestrado. Inmetro, Duque de Caxias, 2016.

TROCHIM, William MK; DONNELLY, James P. Research methods knowledge base. 2001.

WITTMANN, Milton Luiz et al. Uma visão não-linear pelo prisma da complexidade sobre o ambiente da estratégia empresarial. V Encontro de Estudos em Estratégia. Porto Alegre, 2011.. 